\title{
REPRESENTATION OF FUNCTIONS IN $C(X)$ BY MEANS OF EXTREME POINTS
}

\author{
N. T. PECK $^{1}$
}

Let $X$ be a compact metric space. It is known that if $U$ is the closed unit ball of $C_{r}(X)$ (the space of continuous real-valued functions on $X$ under the usual sup norm), a necessary and sufficient condition that $U$ be the closed convex hull of the set of its extreme points is that $X$ be totally disconnected (Bade [1]). It is also known (Phelps [4]) that if $C(X)$ is the space of all continuous complex-valued functions on $X$ under the sup norm, and if $U$ is the closed unit ball of $C(X), U$ is always equal to the closed convex hull of the set of its extreme points (see also Goodner [2]). It is our purpose in this note to obtain information about $U$ in the case of $C(X)$ similar to that obtained for $C_{r}(X)$.

We make the following notational conventions: $D$ will denote the closed unit disc in the complex plane and $B$ will denote the set of points in $D$ of modulus 1 . By $E$ we will mean the set of extreme points of $U$ (the closed unit ball of $C(X)) ; E$ is the set of all elements of $U$ which map $X$ into $B$. The topological dimension of $X$ as defined in Hurewicz and Wallman [3] will be denoted by $\operatorname{dim} X$.

Our theorem now reads as follows:

Theorem. Let $X$ be a compact metric space. Then the following are equivalent:

(1) $\operatorname{dim} X \leqq 1$

(2) $U$ is a subset of the convex hull of $E$.

Proof. We first observe that if $f$ is a continuous map of a topological space $Y$ into $D$ which omits the origin, then there are two continuous maps $f_{1}$ and $f_{2}$ of $Y$ into $B$ such that $f=\left(f_{1}+f_{2}\right) / 2$. We now show that condition (1) implies condition (2). (I am indebted to the referee for strengthening and combining several arguments to give the following proof.)

Let $f$ be in $U$. By Theorem VI.1 of Hurewicz and Wallman, the origin is an unstable value of $f$; by Proposition B of the same section, there is a continuous function $h_{1}$ which omits the origin such that

Received by the editors March 1, 1966.

1 Research was partially supported by the National Science Foundation under Grant NSF-GP-3509. 


$$
\begin{aligned}
& \text { If }|f(x)| \geqq 1 / 3, \text { then } h_{1}(x)=f(x) ; \\
& \text { if }|f(x)|<1 / 3, \text { then }\left|h_{1}(x)\right|<1 / 3 .
\end{aligned}
$$

Put $h_{2}=2 f-h_{1}$. Then it is clear that $h_{1}$ and $h_{2}$ are in $U$.

Suppose $\left|h_{1}(x)\right|>3 \epsilon>0$ for all $x \in X$. By the same results in [3], there is a continuous function $g_{2}$ such that $g_{2}$ omits the origin and such that

$$
\begin{aligned}
& \text { If }\left|h_{2}(x)\right| \geqq \epsilon, \quad \text { then } g_{2}(x)=h_{2}(x) ; \\
& \text { if }\left|h_{2}(x)\right|<\epsilon, \quad \text { then }\left|g_{2}(x)\right|<\epsilon .
\end{aligned}
$$

Put $g_{1}=2 f-g_{2}$. Now it is easy to check that $g_{1}$ and $g_{2}$ are in $U$; moreover $g_{1}$ omits the origin since $\left|g_{1}(x)-h_{1}(x)\right|=\left|g_{2}(x)-h_{2}(x)\right| \leqq 2 \epsilon$ for all $x \in X$. By the remark at the beginning of the proof, $g_{1}$ and $g_{2}$ are in the convex hull of $E$; hence $f=\left(g_{1}+g_{2}\right) / 2$ is in the convex hull of $E$.

We now prove that condition (2) implies condition (1). By [3, Theorem VI, \$4] it suffices to prove the following: Let $C$ be a closed subset of $X$. Then if $f$ is a continuous map of $C$ in to $B$, there is an extension of $f$ to a continuous map of $X$ in to $B$.

Hence, let $C$ and $f$ be as above. Using Tietze's theorem, we can extend $f$ to a continuous $\tilde{f}$ from $X$ in to $D$. If condition (2) holds, there is a probability measure $\mu$ on $U$ (even one with finite support) such that $\mu(E)=1$ and such that $L(\tilde{f})=\int L(g) d \mu(g)$ for all $L$ in the (complex) dual of $C(X)$. Let $\left\{x_{n}\right\}_{n=1}^{\infty}$ be a sequence dense in $C$ and define linear functionals $L_{n}$ on $C(X)$ by $L_{n}(h)=h\left(x_{n}\right)$ for $h$ in $C(X)$. Then for each $n$ we have

$$
\tilde{f}\left(x_{n}\right)=L_{n}(\tilde{f})=\int L_{n}(g) d \mu(g)=\int g\left(x_{n}\right) d \mu(g) ;
$$

we may divide to obtain

$$
1=\int_{E} \frac{g\left(x_{n}\right)}{\tilde{f}\left(x_{n}\right)} d \mu(g) \quad \text { for all } n .
$$

Since $\left|\tilde{f}\left(x_{n}\right)\right|=\left|g\left(x_{n}\right)\right|=1$ for all $g$ in $E$ and since $\mu$ is a probability measure, it must be the case that

$$
\mu\left\{g \in E: g\left(x_{n}\right) \neq \tilde{f}\left(x_{n}\right)\right\}=0 \quad \text { for each } n .
$$

Hence,

$$
\mu\left(\bigcup_{n=1}^{\infty}\left\{g \in E: g\left(x_{n}\right) \neq \tilde{f}\left(x_{n}\right)\right\}\right)=0
$$


it follows that there is a $g^{*}$ in $E$ such that $g^{*}\left(x_{n}\right)=\tilde{f}\left(x_{n}\right)=f\left(x_{n}\right)$ for all $n$; since $\left\{x_{n}\right\}$ is dense in $C, g^{*}(x)=f(x)$ for all $x$ in $C$. This $g^{*}$ is the desired extension of $f$ and the proof is thereby complete.

\section{BIBLIOGRAPHY}

1. W. Bade, Functional analysis seminar notes, University of California, Berkeley, 1957 (unpublished).

2. D. B. Goodner, The closed convex hull of certain extreme points, Proc. Amer. Math. Soc. 15 (1964), 256-258.

3. W. Hurewicz and H. Wallman, Dimension theory, Princeton Univ. Press, Princeton, N. J., 1941.

4. R. R. Phelps, Extreme points in function algebras, Duke Math. J. 32 (1965), 267-277.

YALE UNIVERSITY 